\section{Gene and drug matrix for personalized cancer therapy}

\author{
Tim Harris
}

The recent Perspective by Richard Schilsky (Nature Rev. Drug Discov. 9, 363-367; $\underline{2010})^{1}$ suggests that the 'future is now' for personalized medicine in the treatment of cancer ${ }^{1}$. Examples of genetic analyses that drive clinical decision-making in cancer include the use of imatinib in the treatment of chronic myeloid leukaemia with BCR-ABL translocations; using gefitinib or erlotinib to treat lung cancer with epidermal growth factor receptor (EGFR) mutations; treating human epidermal growth factor receptor 2 (HER2/neu)-positive patients with breast cancer with trastuzumab; and not using EGFR monoclonal antibodies in patients with colon cancer tumours harboring KRAS mutations.

The sequencing of normal DNA and tumour DNA is now common practice. However, there is little use for large-scale genome or exome sequencing of patient and tumour DNA in the clinical environment unless the results can provide guidance to selecting a specific therapy.

The ability to measure changes in genes known to be involved in various cancers at high frequency has made it possible to define tumours by their molecular pathology, as well as their cellular pathology ${ }^{2}$. Several mutant gene profiling sets are being used today in the routine analysis of cancers in the clinic. A group of researchers at Massachusetts General Hospital, USA, have established the Translational Research Laboratory (TRL), which performs a broad genotyping screen in tumours, termed SNAPSHOT (http://www2.massgeneral.org/ pathology/molecularpathology.htm). The Dana-Farber Cancer Institute, USA, has a similar profiling set called Oncomap ${ }^{3}$ and researchers at the Memorial Sloan Kettering Cancer Center, USA, are using Sequenom's gene set. Several different methods are being used to type these mutations including TaqMan PCR assays, Sanger DNA sequencing and mass spectrometry. It will not be long before these traditional methods are superseded by cheaper digital higherthroughput DNA sequencing systems that can measure changes in gene expression and detect mutations at the same time ${ }^{4}$.
As a consequence of these gene profiling studies, physicians are being left with the increasingly complex question of which drugs to use to treat tumours that have one or more of these cancer-associated molecular defects. There are two aspects to this challenge: one is the ability to define the molecular defect or defects in the tumour, and the other is access to available drugs on the market (or in clinical development) that are likely to be appropriate for the treatment of that subclass of disease in the context of other relevant (chemo) therapies.

We considered it worthwhile to compare the common genetic lesions that have been found in various cancers (by both DNA sequencing and other discovery techniques) against the current pharmacopoeia available for their treatment. TABLE 1 shows a matrix of the kinase genes that carry mutations or that are overexpressed in certain types of cancer, plotted against the currently available drugs on the market. At this stage the study has been restricted to genes encoding protein kinases, including receptor tyrosine kinases, and to small molecule compounds. The various monoclonal antibodies that target the EGFR or vascular endothelial growth factor (VEGF) receptors, HER2 or other targets as described in the Perspective by Schilsky have not been included.

Of particular note are the gatekeeper residue mutations in $\mathrm{BCR}-\mathrm{ABL}$, KIT and platelet-derived growth factor receptor (PDGFR), which lead to imatinib resistance and the equivalent EGFR T790M mutation, which causes resistance to erlotinib and geftinib ${ }^{5}$. Therapeutic options available for patients with drug-resistant tumours are important, and new drugs are now being tailored to overcome resistance mutations.

There are other examples of drugs that are currently in development, for which knowledge of the molecular defects in a tumour informs the stratification of patients. The most pertinent examples are PLX4032 for patients with the BRAF V600E mutation in melanoma and the use of crizotinib (a MET inhibitor that blocks

ALK) for the treatment of non-small cell lung carcinoma with ALK translocations or for the treatment of neuroblastoma with ALK-activating mutations or overexpression $^{6,7}$.

Although the numbers are currently small this matrix of 'genes versus drugs' is growing rapidly and will expand dramatically as our understanding of tumour mutations increases and as new inhibitors with different specificities emerge. A more extensive analysis comparing many of the kinase inhibitors in late-stage development against this same set of genes is available on request.

The therapeutic impact of personalized health care utilizing robust diagnostic assays and selected therapies will be considerable. Information such as that provided by TABLE 1 above will be needed to inform oncologists and allow them to treat patients in a more personalized way.

Tim Harris is the Chief Technology Officer at SAIC-Frederick, PO Box B, 1050 Boyles Street, Frederick, Maryland 21702, USA. e-mail: harristjr@mail.nih.gov doi: 10.1038/nrd3181-c1

1. Schilsky, R. Personalized medicine in oncology: the future is now. Nature Rev. Drug Discov. 9, 363-367 (2010)

2. Harris, T. J. R. \& McCormick, F. The molecular pathology of cancer. Nature Rev. Clin. Oncol. 7 251-265 (2010)

3. MacConnaill, L. E. et al. Profiling critical cancer gene mutations in clinical tumour samples. PLoS One 4, e7887 (2009)

4. Munroe, D. J. \& Harris T. J. R. Third generation sequencing fireworks at Marco Island. Nature Biotech. 28, 426-428 (2010).

5. Zhang, J., Yang, P. L. \& Gray, N. S. Targeting cancer with small molecule kinase inhibitors. Nature Rev. Cancer 9, 28-39 (2009).

6. Garber, K. Melanoma drug vindicates targeted approach. Science 326, 1619 (2009)

Mosse, Y. P., Wood, A. \& Maris, J. M. Inhibition of ALK signalling for cancer therapy. Clin. Cancer Res. 15, 5609-5614 (2009).

8. Li, J. et al. A chemical and phosphoproteomic characterisation of dasatanib action in lung cancer Nature Chem. Biol. 6, 291-299 (2010).

9. Bukowski, R. M., Yasothan, U. \& Kirkpatrick, P. Pazopanib. Nature Rev. Drug Discov. 9, 17 (2010).

10. Engelman, J. A. et al. MET amplification leads to gefitinib resistance in lung cancer by activating ERBB3 signalling. Science 316, 1039-1043 (2007).

Acknowledgements

This project has been funded in whole or in part with federal funds from the National Cancer Institute, National Institutes of Health, under Contract No. HHSN261200800001E. The content of this publication does not necessarily reflect the views or policies of the Department of Health and Human Services, nor does mention of trade names, commercial products, or organizations imply endorsement by the US Government.

FURTHER INFORMATION

Entrez Gene: http://www.ncbi.nlm.nih.gov/entrez

The Cancer Genome Atlas: http://cancergenome.nih.gov

The Cancer Genome Project:

http://www.sanger.ac.uk/genetics/CGP

ALL LINKS ARE ACTIVE IN THE ONLINE PDF 


\begin{tabular}{|c|c|c|c|c|c|c|c|c|c|c|}
\hline \multirow[t]{2}{*}{ Gene* } & \multicolumn{10}{|l|}{ Drug $^{\ddagger}$} \\
\hline & Dasatinib $^{\S}$ & Erlotinib & Everolimus & Gefitinib & Imatinib & Lapatinib & Nilotinib & Pazopanib" & Sorafenib & Sunitinib \\
\hline \multicolumn{11}{|l|}{ ALK } \\
\hline \multicolumn{11}{|l|}{$A U R K A / B$} \\
\hline $\begin{array}{l}B C R-A B L \\
(m u t)^{\pi}\end{array}$ & $\checkmark$ & & & & & & $\checkmark$ & & & \\
\hline \multicolumn{11}{|l|}{$\begin{array}{l}\text { BCR-ABL } \\
\text { (T315I) }\end{array}$} \\
\hline$B R A F$ & $\checkmark$ & & & & & & & & $\checkmark$ & \\
\hline \multicolumn{11}{|c|}{ EGFR (T790M) } \\
\hline \multicolumn{11}{|c|}{ FGFR1 } \\
\hline \multicolumn{11}{|l|}{ FGFR3 } \\
\hline \multicolumn{11}{|l|}{ FGFR4 } \\
\hline FLT3 & & & & & & & & & & $\checkmark$ \\
\hline HER2/neu & & & & & & $\checkmark$ & & & & \\
\hline \multicolumn{11}{|l|}{ IGF1 } \\
\hline JAK2 & & $\checkmark$ & & & & & & & & \\
\hline KIT & & & & & $\checkmark$ & & $\checkmark$ & & & $\checkmark$ \\
\hline \multicolumn{11}{|c|}{ P13KCA } \\
\hline \multicolumn{11}{|l|}{ PLK1 } \\
\hline \multicolumn{11}{|l|}{ RET } \\
\hline \multicolumn{11}{|l|}{ MST1R } \\
\hline \multicolumn{11}{|l|}{ RPS6KB1 } \\
\hline VEGFR1 & & & & & & & & $\checkmark$ & $\checkmark$ & $\checkmark$ \\
\hline VEGFR2 & & & & & & & & $\checkmark$ & $\checkmark$ & $\checkmark$ \\
\hline
\end{tabular}

AURK, Aurora kinase; CDK5, cyclin-dependent kinase 5; EGFR, epidermal growth factor receptor; FGFR, fibroblast growth factor receptor; HER, human epidermal growth factor receptor (also known as ERBB2); IGF, insulin-like growth factor; JAK, janus kinase; PDGFR, platelet-derived growth factor receptor; PI3KCA, phosphoinositide-3-kinase catalytic subunit alpha; PLK1, polo-like kinase 1; VEGFR1, vascular endothelial growth factor receptor 1 (also known as FLT1); VEGFR2, vascular endothelial growth factor receptor 2 (also known as KDR). The ticks refer to those kinases that are inhibited by the relevant drug. ${ }^{\star}$ The genes were selected from a variety of sources including REFS 2,5 and http://www.sanger.ac.uk/genetics/CGP/cosmic (version 46). ₹See the following website for more information about the drugs: http://www.cancer.gov/drugdictionary/. ${ }^{.}$Dasatanib has a broad specificity inhibiting SRC family members and ephrin receptors ${ }^{8} . "$ see REF 9. "BRC-ABL (mut) mutants refers to all imatinib-resistant mutants of BCR-ABL except T315I. "MET amplification also leads to gefitinib resistance ${ }^{10}$. 